\title{
Measles among healthcare workers: a potential for
}

\section{nosocomial outbreaks}

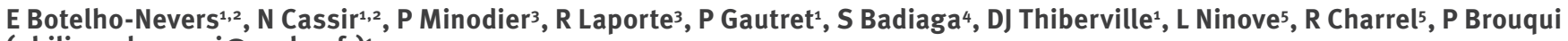
(philippe.brouqui@ap-hm.fr) ${ }^{1}$

1. Pôle de Maladies Infectieuses, IFR 48, Assistance Publique Hôpitaux de Marseille, France

2. These two authors contributed equally to this work

3. Pôle mère-enfants, Assistance Publique Hôpitaux de Marseille, France

4. Pôle AUR, Assistance Publique Hôpitaux de Marseille, France

5. Laboratoire de virologie, IFR 48, Assistance Publique Hôpitaux de Marseille, France

Citation style for this article:

Botelho-Nevers E, Cassir N, Minodier P, Laporte R, Gautret P, Badiaga S, Thiberville D, Ninove L, Charrel R, Brouqui P. Measles among healthcare workers: a potential for nosocomial outbreaks. Euro Surveill. 2011;16(2):pii=19764. Available online: http://www.eurosurveillance.org/ViewArticle.aspx?Articleld=19764

Article published on 13 January 2011

We report here 14 cases of measles among healthcare workers (HCWs) in Public Hospitals of Marseilles, France that occurred between April and November 2010. All cases but one were under 30 years of age. Following the identification of these cases, we checked the immune status among $154 \mathrm{HCWs}$ who volunteered to take part in the study and showed that $93 \%$ and $88 \%$ were immune against measles and mumps respectively. HCWs non-immunised against measles were all under 30 years of age.

\section{Introduction}

France has been experiencing a measles outbreak since 2008. A recrudescence of cases among children and young adults has been related to insufficient vaccine coverage [1]. The risk of acquiring measles in non-immune healthcare workers (HCWs) is estimated to be 13 times higher than in general population [2]. Consequently, young non-immune HCWs are highly at risk occupational measles and when infected constitute a risk of transmission to non-immune or immunocompromised patients [3-5]. Indeed measles is a highly contagious disease [6]; therefore, strict adherence to alcohol-based hand rub and rapid implementation of appropriate respiratory isolation measures are essential but insufficient to prevent measles outbreaks in hospital settings [7]. Vaccination is consequently the only reliable protection against nosocomial spread of measles [3]. In France, five vaccines are mandatory for HCWs: vaccines against diphtheria, tetanus, poliomyelitis, hepatitis $B$ virus, and tuberculosis. Other vaccines such as the vaccine against measles are only recommended [8]. According to the recently released national guidelines regarding measles vaccination for HCWs, getting vaccinated against measles or completing measles vaccination is recommended but not mandatory [9]. Few reports described seroprevalence against measles among HCWs in general: a high level of immunity has been reported in Italy and the United Kingdom $[10,11]$. However, it has been described to be lower among nurse and medical students in Switzerland [12]. The last European measles serosurvey including French data on different age groups was published in 2001 [13].

In 2010, at total of 122 cases of measles were managed in the three of Public Hospitals of Marseilles (PHM) and since April 2010, cases of measles appeared among HCWs of the PHM. Following notification of the first case, we evaluated the immune status among the HCWs who volunteered to participate. We describe here the measles cases that occurred among HCWs from April 2010 to November 2010 and the immunity against measles of 154 volunteer HCWs working in three wards at high risk for transmission of contagious diseases (such as infectious disease, emergency room, paediatric, maternity and oncology wards). In the same period, a cluster of three mumps cases occurred among medical students at the associated School of Medicine and therefore we also checked the immune status for mumps of the $154 \mathrm{HCWs}$.

\section{Identification of measles cases}

Measles cases among the HCWs of the PHM were identified in three different ways: through (i) the infectious diseases specialist of PHM (6 cases), (ii) the occupational medicine or infection control unit (5 cases), (iii) the laboratory database of the hospital (3 cases) that contained information for all HCWs of PHM for the period from January to November 2010.

PHM has approximately 15,000 staff members including all statutory personnel and medical and nurse students.

\section{Seroprevalence of IgG against} measles and mumps in HCWs

Between April and November 2010, all HCWs $(n=363)$ of the infectious disease ward, the paediatric and the adult emergency rooms of North Marseille Hospital one of the three locations of PHM were invited to participate in a study aimed to clarify their immune status against measles and mumps. A short questionnaire recording occupation, age and history of measles and mumps 
immunisation or past infection was distributed to the participants. Answers to questions were collected in Excel, frequencies, means and univariate analysis were performed with Epilnfo version 3.5.1 August 2008 (Centers for Disease Prevention and Control, Atlanta, USA). HCWs who accepted to participate in the study were invited to have a blood test for measles and mumps and to sign a written consent to take part in the study. HCWs were screened for measles and mumps IgG on serum sample by enzyme-linked immuno-sorbent assay (ELISA) (Siemens, France).Test was considered positive for measles and mumps if antibody titers were above $500 \mathrm{mIU} / \mathrm{mL}$. HCWs with negative measles or mumps IgG were informed about the result and were offered immunisation with either a measles vaccine or measles-mumps-rubella (MMR) vaccine according

\section{FIGURE 1}

Measles cases among healthcare workers and patients of Public Hospitals of Marseilles, France, January -

November 2010

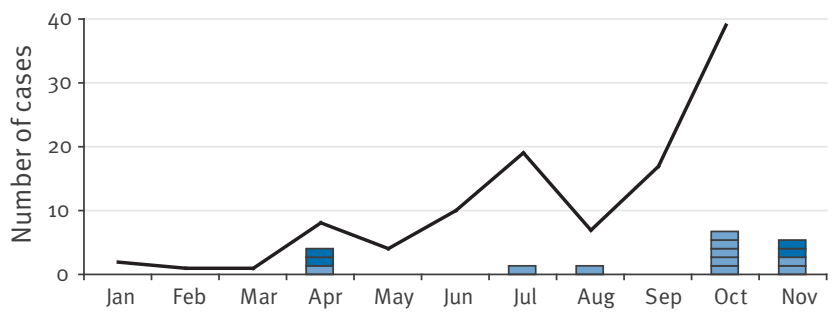

The black curve represents measles cases among patients.

Each box represents one measles case among healthcare workers. Dark blue boxes represent related measles cases among healthcare workers. to availability of vaccine and sex (MMR vaccines were used for women).

\section{Results}

Measles cases among healthcare workers

Fourteen laboratory-confirmed measles cases occurred among HCWs at PHM. The mean age was 27.54 +/- 4.70 years (range 22-39) and nine were women. The approximate attack rate of measles was of 93 cases per 100,000 HCWs. Ten cases occurred in medical staff: five were residents, three were medical students and two were medical doctors. The four remaining cases occurred in two nurses and two nurse assistants. Distribution of cases among HCWs and patients of PHM during the year 2010 is presented in Figure 1.

During the same period, 108 cases of measles were diagnosed at our institution among patients.

To the best of our knowledge, no transmission from HCWs to patients occurred. Measles vaccination status was available for 10 cases: six HCWs were unvaccinated and four had received only one dose of measles-containing vaccine in childhood. Place of infection was considered the hospital for 12 cases (certain in eight cases (i.e. HCWs working in a ward where cases of measles in patients have been managed in the previous 15 days), probable in four cases (i.e, HCWs with no direct contact with patients infected with measles but contact with HCWs managing these patients) and the community for two cases. Two cases acquired despite the post-exposure measles vaccination performed in 48 hours.

\section{TABLE 1}

Immune status for measles and mumps by occupation and age among healthcare workers participating in the study, Public Hospitals of Marseilles, France, April - November $2010(\mathrm{n}=154)$

\begin{tabular}{|l|c|c|c|}
\hline Occupation & Number (\%) & Number of HCWs immune to measles (\%) & Number of HCWs immune to mumps (\%) \\
\hline Medical Doctor & $19(12)$ & $19(100)$ & $18(95)$ \\
\hline Resident & $19(12)$ & $18(95)$ & $47(84)$ \\
\hline Medical student & $55(36)$ & $50(91)$ & $28(87)$ \\
\hline Nurse & $32(21)$ & $30(94)$ & $25(93)$ \\
\hline Nurse assistant & $27(17)$ & $25(93)$ & $2(100)$ \\
\hline Other & $2(1)$ & $2(100)$ & $43(83)$ \\
\hline Age (years) & & & $30(88)$ \\
\hline $19-24$ & $52(34)$ & $45(86)$ & $14(93)$ \\
\hline $25-29$ & $34(22)$ & $31(91)$ & $11(92)$ \\
\hline $30-34$ & $15(10)$ & $15(100)$ & $11(92)$ \\
\hline $35-39$ & $12(8)$ & $12(100)$ & $12(92)$ \\
\hline $40-44$ & $12(8)$ & $12(100)$ & $8(89)$ \\
\hline $45-49$ & $13(8)$ & $13(100)$ & $6(100)$ \\
\hline $50-54$ & $9(6)$ & $9(100)$ & $1(100)$ \\
\hline $55-59$ & $6(4)$ & $6(100)$ & $136(88)$ \\
\hline $60-65$ & $1(1)$ & $1(100)$ & \\
\hline Total & 154 & $144(93)$ & \\
\hline
\end{tabular}

HCW: healthcare worker. 
Serosurvey in healthcare workers at the Public Hospitals of Marseilles

A total of $154 \mathrm{HCWs}$ took part in the study, representing a participation rate of $42.4 \%(154 / 363) ; 74$ in the infectious diseases department, 57 and 23 in the paediatric and the adult emergency rooms respectively. All 154 HCWs answered to the questionnaire and had blood tests for measles and mumps. The breakdown of participants by occupation and age are shown in Table 1 .

The mean age of the participating HCWs was 32.4 years +/- 11.1 (range $19-65$ years), 118 were women ( $76 \%$ ). Of the $154 \mathrm{HCWs}, 144(93 \%)$ and $136(88 \%)$ had a positive IgG serology for measles and mumps respectively. The HCWs in the age groups of 19-24 and 25-29 years had a seroprevalence of $86.5 \%$ and $91.2 \%$ respectively (Figure 2).

The absence of immunity against measles (naturally acquired or through vaccination) was significantly associated with younger age groups (mean age 23.9 $+/-2.4$ years for non-immune HCWs vs. $32.9+/-11.3$ years for immune HCWs, $p=0.011$ )

Around a quarter and a third of the HCWs did not know their immune status for measles and mumps, respectively (Table 2). The number of vaccine doses was often unknown among vaccinated HCWs.

\section{Discussion}

Our study reports a series of measles cases among healthcare workers during 2010. Measles spreads in Europe and in France with large outbreaks among general population since 2008 but an increase in the number of cases was noticed in 2010 in France [1].

\section{FIGURE 2}

Healthcare workers with immunity against measles by age group and occupation, Public Hospitals of Marseilles, France, April - November 2010

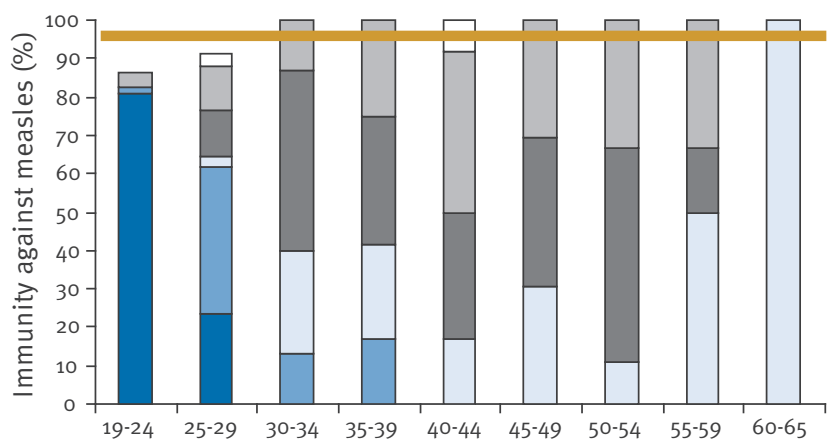

Age group (years)

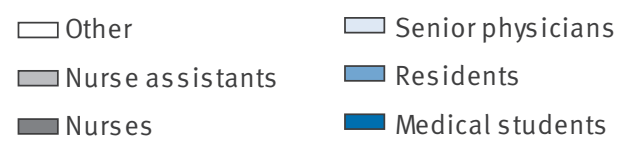

The age groups of $19-24$ and $25-29$ years had a seroprevalence of $86.5 \%$ and $91.2 \%$ respectively.

The orange line represents the target country-wide vaccination coverage (95\%) suggested by the World Health Organization for the elimination of the disease [14].

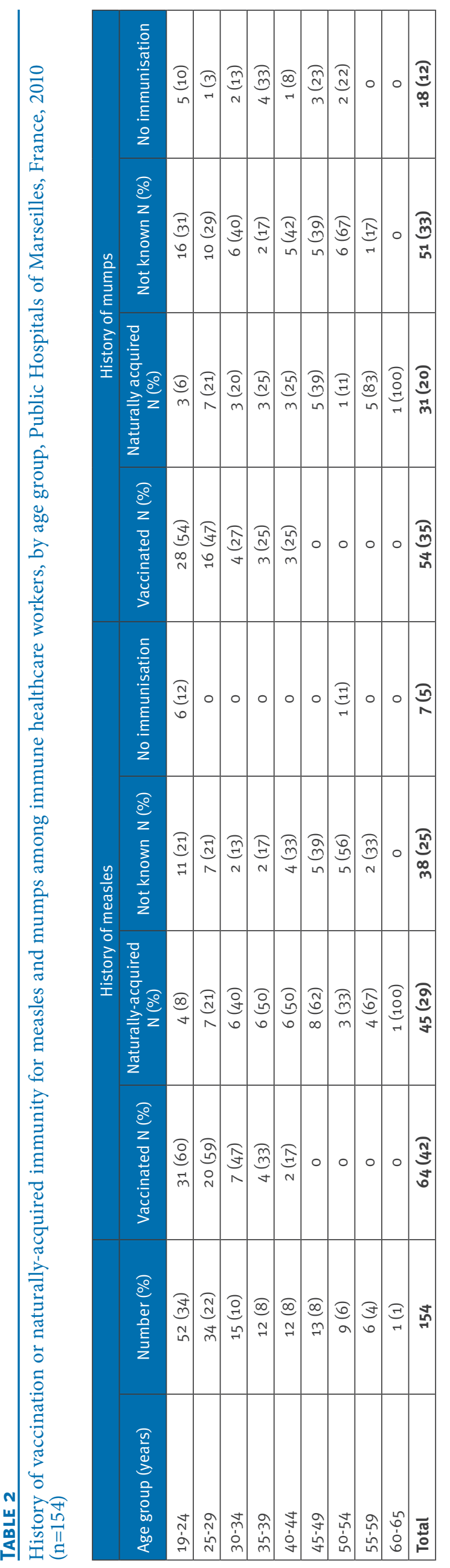


Marseilles is one of the areas in France that experienced a high incidence of measles in 2010, with an incidence of 14.8 per 100,000 population [15]. The incidence of measles among HCWs in PHM seems much higher than in the general population. One can assume that the identification of measles cases among HCWs is more exhaustive than in the general population where the incidence is largely underestimated [15]. However it cannot be excluded that measles cases among HCWs may have been missed. The high attack rate of measles among HCWs presented here indicates the high risk for transmission of measles in healthcare settings among non-immune persons $[1-3,16,17]$. In our study as in the general population, cases of measles affected mainly young adults aged between 20 and 30 years $[1,15]$ who had not been vaccinated against measles or who had received only one dose of measles-containing vaccine. Our report shows that at least eight measles cases among HCWs would have been prevented if national guidelines had been applied [9]. Although the selection of HCWs on a voluntary basis may have introduced a bias in the participation rate, the sample selected here remains representative for PHM staff. However, the data may be extrapolated only to teaching hospitals in France where young students and HWCs are usually employed. Occupational medicine and infection control unit checked the immune status of staff and patients exposed and suggested post-exposure prophylaxis when necessary as recommended in the national guidelines [9]. In this outbreak, post-exposure vaccination performed in the 72 hours after exposure as recommended $[8,9]$ failed to prevent measles in two cases. Therefore, all susceptible exposed HCW had to stay at home even if prophylaxis measures were undertaken. While post-exposure prophylaxis (immune globulines) had been given for immunocompromised patients [9], transmission to patients could not been excluded notably due to prolonged incubation period of measles.

Our seroprevalence study revealed that $6.5 \%$ of HCWs participating in the study were susceptible to measles. All susceptible HCWs were younger than 30 years with a significant association between susceptibility to measles and younger age but no link could be established with the HCW occupation. This observation confirms the need to focus the attention on high-risk age groups among HCWs. Susceptibility for mumps is 11.7 $\%$ among HCWs and is also higher in younger HCWs but with no significant difference among age groups. This susceptibility to measles and mumps among younger population is due to a suboptimal vaccination coverage with often a single vaccine dose [1] compared to adults older than 30 years that had nearly all acquired natural immunisation [13]. Our results on susceptibility to measles and mumps among HCWs are similar to those found in the literature from other European countries [10,11]. Moreover, as described elsewhere [18], we showed that at least a fourth of the HCWs do not know their immune status for measles and mumps. Therefore, in our opinion, each HCW, irrespective of their occupation, younger than 30 years should be tested for measles antibodies. All susceptible HCWs should be promptly vaccinated. Vaccination of all susceptible HCWs should be implemented in hospitals by the occupational medical staff during the medical check-up before recruitment but also by preventive medical staff before enrolling in the school of medicine or in schools for nurses. As immunisation remains the only reliable protection against the spread of measles [3] we suggest HCWs refusing vaccination should be deferred from caring for immunocompromised patients.

\section{Acknowledgements}

We are grateful to our residents Honorat Estelle, Vernier Mireille and Bonardo Anne-Sophie for their help to collect the data.

\section{References}

1. Parent du Châtelet I, Antona D, Freymuth F, Muscat M, Halftermeyer-Zhou F, Maine C, et al. Spotlight on measles 2010: Update on the ongoing measles outbreak in France, 2008-2010. Euro Surveill. 2010;15(36):pii=19656. Available from: http://www.eurosurveillance.org/ViewArticle. aspx?Articleld=19656

2. Williams WW, Preblud SR, Reichelderfer PS, Hadler SC. Vaccines of importance in the hospital setting. Problems and developments. Infect Dis Clin North Am. 1989;3(4):701-22.

3. Botelho-Nevers E, Chevereau L, Brouqui P. Letter to the editor. Spotlight on measles 2010: Measles in healthcare workers - vaccination should be revisited. Euro Surveill. 2010;15(41): pii=19687. Available from: http://www. eurosurveillance.org/ViewArticle.aspx?Articleld=19687

4. Rivera ME, Mason WH, Ross LA, Wright HT Jr. Nosocomial measles infection in a pediatric hospital during a communitywide epidemic. J Pediatr. 1991;119(2):183-6.

5. Steingart KR, Thomas AR, Dykewicz CA, Redd SC. Transmission of measles virus in healthcare settings during a communitywide outbreak. Infect Control Hosp Epidemiol 1999;20(2):115-9.

6. Mossong J, Muller CP. Estimation of the basic reproduction number of measles during an outbreak in a partially vaccinated population. Epidemiol Infect. 2000;124(2):273-8.

7. Bolyard EA, Tablan OC, Williams WW, Pearson ML, Shapiro CN, Deitchmann SD. Guideline for infection control in healthcare personnel, 1998. Hospital Infection Control Practices Advisory Committee. Infect Control Hosp Epidemiol. 1998;19(6):407-63.

8. Institut de Veille Sanitaire (InVS, French Institute for Public Health Surveillance). Vaccination schedule and recommendations from the "Haut conseil de la santé publique" in France. BEH. $2010 ; 14-15$. Available from: http://www.invs. sante.fr/beh/2010/14_15/beh_14_15.pdf

9. Ministère de la santé et des sports. CIRCULAIRE NDGS/ $\mathrm{RI} 1 / 2009 / 334$ du 4 novembre 2009 relative à la transmission obligatoire de données individuelles à l'autorité sanitaire en cas de rougeole et la mise en oeuvre de mesures préventives autour d'un cas ou de cas groupés [Circular No. DGS/RI1/2009/334 of 4 November 2009 on the mandatory notification of individual data to the health authority in case of measles and implementation of preventive measures around a case or a cluster]. French. Available from: http://www.sante. gouv.fr/IMG/pdf/o9_334topdf.pdf

10. Fedeli U, Zanetti C, Saia B. Susceptibility of healthcare workers to measles, mumps rubella and varicella. J Hosp Infect. 2002;51(2):133-5.

11. Ziegler E, Roth C, Wreghitt T. Prevalence of measles susceptibility among health care workers in a UK hospital. Does the UK need to introduce a measles policy for its health care workers? Occup Med (Lond). 2003;53(6):398-402.

12. Baer G, Bonhoeffer J, Schaad UB, Heininger U. Seroprevalence and immunization history of selected vaccine preventable diseases in medical students. Vaccine. 2005;23(16):2016-20.

13. de Melker H, Pebody RG, Edmunds WJ, Levy-Bruhl D, Valle M, Rota MC, et al. The seroepidemiology of measles in Western Europe. Epidemiol Infect. 2001;126(2):249-59. 
14. World Health Organization (WHO). Strategic plan for measles and congenital rubella infection in the WHO European Region. Copenhagen, Denmark, WHO Regional Office for Europe; 2003. Available from: http://www.euro.who.int/document/e81567.pdf

15. Six C, Blanes de Canecaude J, Duponchel JL, Lafont E,

Decoppet A, Travanut M, et al. Spotlight on measles 2010: Measles outbreak in the Provence-Alpes-Côte d'Azur region, France, January to November 2010 - substantial underreporting of cases. Euro Surveill. 2010;15(50):pii=19754. Available from: http://www.eurosurveillance.org/ViewArticle. aspx?Articleld $=19754$

16. Tafuri S, Germinario C, Rollo M, Prato R. Occupational risk from measles in healthcare personnel: a case report. J Occup Health 2009;51(1):97-9.

17. Grgic-Vitek M, Frelih T, Ucakar V, Prosenc K, Tomažič J, Petrovec M, et al. Spotlight on measles 2010: A cluster of measles in a hospital setting in Slovenia, March 2010. Euro Surveill. 2010;15(20):pii=19573. Available from: http://www. eurosurveillance.org/ViewArticle.aspx?Articleld=19573

18. Loulergue P, Moulin F, Vidal-Trecan G, Absi Z, Demontpion $C$, Menager $C$, et al. Knowledge, attitudes and vaccination coverage of healthcare workers regarding occupational vaccinations. Vaccine. 2009;27(31):4240-3 